\title{
Correlation analysis of IL-4, IL-10 and APN levels with postoperative infection of colorectal cancer
}

\author{
JIANYUN HE ${ }^{1 *}$, ZHIYAN WANG ${ }^{2 *}$ and SIHAI ZHANG ${ }^{2}$ \\ ${ }^{1}$ Cross Infection Control Office, Xiangyang No. 1 People's Hospital, Hubei University of Medicine, Xiangyang, Hubei 441000; \\ ${ }^{2}$ Department of General Surgery, Ningbo Yinzhou No. 2 Hospital, Ningbo, Zhejiang 315000, P.R. China
}

Received August 27, 2018; Accepted November 30, 2018

DOI: 10.3892/ol.2018.9798

\begin{abstract}
Changes in expression levels of serum interleukin-4 (IL-4), IL-10 and adiponectin (APN) in patients with postoperative infection of colorectal cancer were studied. The clinical data of 159 patients receiving radical surgery for colorectal cancer in Xiangyang No. 1 People's Hospital, Hubei University of Medicine from January 2014 to December 2017, were retrospectively analyzed. A total of 67 patients with postoperative infection were enrolled into the infection group, while the remaining 92 patients without infection were enrolled into the non-infection group. The expression levels of serum IL-4, IL-10 and APN of patients were detected via enzyme-linked immunosorbent assay. The correlation of IL-4, IL-10 and APN levels with stage of colorectal cancer were explored by the Spearman's correlation analysis. The expression levels of IL-4 and IL-10 in the infection group were significantly higher than those in the non-infection group at day 3 after surgery $(\mathrm{P}<0.05)$. The expression level of APN in the infection group was lower than that in the non-infection group at day 3 after surgery $(\mathrm{P}<0.05)$. The serum IL-4 and IL-10 levels in pulmonary infection was higher and the serum IL-10 level in pulmonary infection was higher than those in incision infection and abdominal infection $(\mathrm{P}<0.05)$. The IL-4 and IL-10 levels in patients with colorectal cancer in the infection group at day 3 after surgery had a significant positive correlation with the stage of colorectal cancer $(r=0.9357$, $\mathrm{P}<0.001 ; \mathrm{r}=0.9717, \mathrm{P}<0.001)$, and the APN level in patients with colorectal cancer in the infection group at day 3 after surgery had a significant negative correlation with the stage of colorectal cancer $(r=-0.9736, \mathrm{P}<0.001)$. The serum IL-4 and IL-10 levels in patients with postoperative infection of
\end{abstract}

Correspondence to: Dr Sihai Zhang, Department of General Surgery, Ningbo Yinzhou No. 2 Hospital, 998 Qian Hebei Road, Ningbo, Zhejiang 315000, P.R. China

E-mail: re656d@163.com

*Contributed equally

Key words: IL-4, IL-10, APN, postoperative infection of colorectal cancer, stage of colorectal cancer, correlation colorectal cancer are positively correlated with the stage of cancer, while the serum APN level was negatively correlated with the stage of cancer.

\section{Introduction}

Colorectal cancer is a malignant tumor of the digestive tract derived from mucous epithelium, including colon cancer and rectal cancer (1). With the changes in dietary structure and living habits, the incidence rate of colorectal cancer ranks $3^{\text {rd }}$ following lung cancer and breast cancer, and its mortality rate shows a gradual increasing trend (2). The 5-year survival rate of early colorectal cancer can be up to $90 \%$, so the early diagnosis and treatment is extremely important (3). Radical surgery dominates the treatment of early colorectal cancer, supplemented by chemotherapy. Patients who can tolerate surgery with middle-advanced cancer and surgical indications should undergo radical surgery. Good efficacy has been obtained in most patients who have received radical surgery for colorectal cancer (4). However, radical surgery for colorectal cancer is difficult, and the patient's immunity is low, so postoperative infection occurs easily (5). The risk of infection is increased due to the decline in the patient's defense after surgery, and incision, pulmonary and abdominal infections often occur after exogenous or endogenous surgical site infection (6).

Interleukin-4 (IL-4) and IL-10 can activate and regulate immune cells, and mediate the activation, proliferation and differentiation of $\mathrm{T}$ and $\mathrm{B}$ cells, which play an important role in inflammatory and immune responses (7). Adiponectin (APN) is an insulin-sensitizing hormone, its level can predict the development of inflammation and it has displayed anti-inflammatory potential in clinical tests (8). Studies have demonstrated that inflammation is involved in the occurrence and development of postoperative infection, and there are significant changes in the levels of serum IL-4, IL-10 and APN, which can evaluate the postoperative infection and serve as indexes for the clinical auxiliary diagnosis of postoperative infection of malignant tumor (9). Currently, the correlation of changes in serum IL-4, IL-10 and APN levels with postoperative infection of colorectal cancer are rarely reported. In this experiment, the data of patients with infection after radical surgery for colorectal cancer were retrospectively analyzed, the levels of serum IL-4, IL-10 and APN were compared in patients with postoperative infection of colorectal cancer and 
pulmonary infection, and their correlation with the stage of colorectal cancer in patients with postoperative infection were analyzed and explored, so as to provide references for the clinical nursing and treatment after radical surgery for colorectal cancer.

\section{Patients and methods}

Data of patients. The clinical data of 159 patients receiving radical surgery for colorectal cancer in Xiangyang No. 1 People's Hospital, Hubei University of Medicine (Xiangyang, China) from January 2014 to December 2017 were retrospectively analyzed. A total of 67 patients with postoperative infection were enrolled into the infection group, including 39 males and 28 females aged 22-76 years with an average age of $42.38 \pm 6.94$ years, while the remaining 92 patients without infection were enrolled into the non-infection group, including 54 males and 38 females aged 19-71 years with an average age of $39.24 \pm 5.31$ years. To ensure the accuracy and reliability of experimental results, clinical data of patients were compared between the two groups, and there were no significant differences $(\mathrm{P}>0.05)$, proving that they were comparable between the two groups. Basic data of patients are shown in Table I.

Exclusion and inclusion criteria. Inclusion criteria: patients whose examination results of pathological section were in line with the manifestations of colorectal cancer, patients aged above 18 years, patients who were not treated in other hospitals, and patients who underwent radical surgery for colorectal cancer in Xiangyang No. 1 People's Hospital Affiliated to Hubei University of Medicine. Exclusion criteria: patients who did not cooperate in related diagnosis and treatment, pregnant or lactating patients, patients with other diseases that may be related to such cytokine expression and changes, patients with genetic diseases, patients with tumors other than colorectal cancer, or patients with communication disorders or cognitive disorders. The subjects or their families signed the informed consent and cooperated with medical workers in related diagnosis and treatment.

This study was approved by the Ethics Committee of Xiangyang No. 1 People's Hospital, Hubei University of Medicine (Xiangyang, China).

Methods. After $4 \mathrm{ml}$ fasting peripheral venous blood was drawn from all patients in the early morning before surgery and at day 3 after surgery, the serum was isolated via centrifugation at $2,000 \mathrm{x} \mathrm{g}$ for $15 \mathrm{~min}$ at $4^{\circ} \mathrm{C}$ (Beckman Coulter, Inc., Brea, CA, USA) and stored in a cryogenic refrigerator (Thermo Fisher Scientific, Inc., Waltham, MA, USA) at $-20^{\circ} \mathrm{C}$. The levels of serum IL-4, IL-10 and APN in both groups were detected via enzyme-linked immunosorbent assay (ELISA) in strict accordance with instructions of the human IL-4 and IL-10 ELISA kits (Shanghai Kanglang Biological Technology Co., Ltd., Shanghai, China) and human APN ELISA kit (Shanghai MLbio Co., Ltd., Shanghai, China). The standard, blank and sample wells were set. Then, $50 \mu 1$ standard samples were accurately loaded, and the sample well was added with $40 \mu \mathrm{l}$ of sample diluent and then $10 \mu \mathrm{l}$ of sample to be detected and mixed evenly. The plate was sealed with sealing membrane, followed by incubation at $37^{\circ} \mathrm{C}$ for $1 \mathrm{~h}$. The sealing membrane was uncovered. The solution was discarded and the plate was dried. Each well was filled with $100 \mu$ l washing solution. The sealing membrane was covered and the washing solution was discarded after $30 \mathrm{sec}$. The above procedure was repeated 5 times, and the plate was dried. The standard well and sample well were added with $100 \mu \mathrm{l}$ ELISA reagent. Color developing agents $\mathrm{A}(60 \mu \mathrm{l})$ and $\mathrm{B}(60 \mu \mathrm{l})$ were added into each well and mixed evenly, and the sealing membrane was covered, followed by color development in the dark at $37^{\circ} \mathrm{C}$ for $30 \mathrm{~min}$. Then $50 \mu \mathrm{l}$ stop buffer was added into each well to terminate the reaction. The optical density (OD) of each well was immediately detected at a wavelength of $450 \mathrm{~nm}$ by using a microplate reader (BioTek Instruments, Inc., Winooski, VT, USA), and the concentrations of serum IL-4, IL-10 and APN were calculated.

Statistical analysis. SPSS 17.4 [AsiaAnalytics (formerly SPSS China)] software system was used for statistical analysis. The basic enumeration data of patients were expressed as percentage [n (\%)], and Chi-square test was performed. IL-4, IL-10 and APN levels were expressed as mean \pm standard deviation. t-test was adopted for the comparison of differences between the two groups at different time-points, and $\mathrm{F}$ analysis was used for the expression difference among different types of infection. The correlation of IL-4, IL-10 and APN levels with the stage of colorectal cancer in patients with postoperative infection of colorectal cancer was analyzed via Spearman's correlation analysis. $\mathrm{P}<0.05$ was considered to indicate a statistically significant difference.

\section{Results}

Changes in expression levels of serum IL-4, IL-10 and APN in both groups. There were no differences in the expression levels of IL-4, IL-10 and APN between the two groups before surgery $(\mathrm{P}>0.05)$. The expression levels of IL- 4 and IL-10 in the infection group were significantly higher than those in the non-infection group at day 3 after surgery $(\mathrm{P}<0.05)$, and they were higher in both groups after surgery than those before surgery $(\mathrm{P}<0.05)$, but the changes in the expression levels in the infection group were more significant than those in the non-infection group. There was no difference in the expression level of APN between the two groups before surgery ( $>0.05$ ). The expression level of APN in the infection group was lower than that in the non-infection group at day 3 after surgery $(\mathrm{P}<0.05)$, and it was lower in both groups after surgery than that before surgery $(\mathrm{P}<0.05)$, but the change in the expression level was more significant in the infection group than that in the non-infection group (Table II).

Expression levels of serum IL-4, IL-10 and APN in different types of infection. The serum IL-4 level in pulmonary infection was higher than that in incision infection and abdominal infection $(\mathrm{P}<0.05)$, and it was also higher in abdominal infection than that in incision infection, displaying no statistically significant difference $(\mathrm{P}>0.05)$. The serum IL-10 level in pulmonary infection was higher than that in incision infection and abdominal infection, showing statistically significant differences $(\mathrm{P}<0.05)$, and it was also higher in 
Table I. Basic data of 159 patients receiving radical surgery for colorectal cancer [n (\%)].

\begin{tabular}{|c|c|c|c|c|}
\hline Parameters & Infection group $(n=67)$ & Non-infection group $(\mathrm{n}=92)$ & $\chi^{2}$ & P-value \\
\hline Sex & & & 0.004 & 0.951 \\
\hline Male & $39(58.21)$ & $54(58.70)$ & & \\
\hline Female & $28(41.79)$ & $38(41.30)$ & & \\
\hline Age (years) & & & 0.070 & 0.791 \\
\hline$<30$ & $27(40.30)$ & $39(42.39)$ & & \\
\hline$\geq 30$ & $40(59.70)$ & $53(57.61)$ & & \\
\hline Smoking & & & 0.222 & 0.637 \\
\hline Yes & $42(62.69)$ & $61(66.30)$ & & \\
\hline No & $25(37.31)$ & $31(33.70)$ & & \\
\hline Dietary habit & & & 2.390 & 0.122 \\
\hline Low fiber & $19(28.36)$ & $37(40.22)$ & & \\
\hline High fiber & $48(71.64)$ & $55(59.78)$ & & \\
\hline Intestinal obstruction & & & 0.164 & 0.686 \\
\hline Yes & $50(74.63)$ & $66(71.74)$ & & \\
\hline No & $17(25.37)$ & $26(28.26)$ & & \\
\hline Surgical mode & & & 0.258 & 0.611 \\
\hline Laparoscopic surgery & $42(62.69)$ & $54(58.70)$ & & \\
\hline Traditional laparotomy & $25(37.31)$ & $38(41.30)$ & & \\
\hline Type of tumor & & & 0.421 & 0.810 \\
\hline Right hemicolon cancer & $15(22.39)$ & $21(22.83)$ & & \\
\hline Left hemicolon cancer & $19(28.36)$ & $22(23.91)$ & & \\
\hline Rectal cancer & $33(49.25)$ & $49(53.26)$ & & \\
\hline Pathological stage & & & 0.272 & 0.965 \\
\hline Stage I & $17(25.37)$ & $23(25.00)$ & & \\
\hline Stage II & $19(28.36)$ & $26(28.26)$ & & \\
\hline Stage III & $24(35.82)$ & $31(33.70)$ & & \\
\hline Stage IV & $7(10.45)$ & $12(13.04)$ & & \\
\hline Type of infection & & & - & - \\
\hline Incision infection & $39(58.21)$ & - & & \\
\hline Abdominal infection & $15(22.39)$ & - & & \\
\hline Pulmonary infection & $13(19.40)$ & - & & \\
\hline
\end{tabular}

Table II. Changes in expression levels of serum IL-4, IL-10 and APN.

\begin{tabular}{|c|c|c|c|c|c|c|}
\hline \multirow[b]{2}{*}{ Groups } & \multicolumn{2}{|c|}{ IL-4 (pg/l) } & \multicolumn{2}{|c|}{ IL-10 (ng/l) } & \multicolumn{2}{|c|}{$\mathrm{APN}(\mathrm{pg} / \mathrm{l})$} \\
\hline & $\begin{array}{l}\text { Before } \\
\text { surgery }\end{array}$ & $\begin{array}{l}3 \text { d after } \\
\text { surgery }\end{array}$ & $\begin{array}{l}\text { Before } \\
\text { surgery }\end{array}$ & $\begin{array}{l}3 \text { d after } \\
\text { surgery }\end{array}$ & $\begin{array}{l}\text { Before } \\
\text { surgery }\end{array}$ & $\begin{array}{l}3 \text { d after } \\
\text { surgery }\end{array}$ \\
\hline $\begin{array}{l}\text { Infection group } \\
(n=67)\end{array}$ & $162.78 \pm 13.76$ & $357.64 \pm 16.48^{a}$ & $153.67 \pm 29.78$ & $219.41 \pm 52.97^{\mathrm{a}}$ & $146.50 \pm 37.53$ & $107.26 \pm 26.61^{\mathrm{a}}$ \\
\hline $\begin{array}{l}\text { Non-infection group } \\
(n=92)\end{array}$ & $159.43 \pm 12.84$ & $216.73 \pm 13.51^{\mathrm{a}}$ & $154.91 \pm 28.56$ & $174.43 \pm 37.65^{\mathrm{a}}$ & $145.92 \pm 36.26$ & $129.12 \pm 29.68^{\mathrm{a}}$ \\
\hline $\mathrm{t}$ & 0.117 & 59.16 & 0.791 & 6.261 & 0.922 & 4.787 \\
\hline P-value & 1.576 & $<0.001$ & 0.266 & $<0.001$ & 0.098 & $<0.001$ \\
\hline
\end{tabular}

aStatistically significant difference compared with that before surgery $(\mathrm{P}<0.05)$. IL, interleukin; APN, adiponectin.

abdominal infection than that in incision infection without a statistically significant difference $(\mathrm{P}>0.05)$. The serum APN level in pulmonary infection was lower than that in incision infection and abdominal infection, showing statistically 
Table III. Changes in expression levels of serum IL-4, IL-10 and APN in different types of infection.

\begin{tabular}{|c|c|c|c|c|c|}
\hline Items & Incision infection $(\mathrm{n}=39)$ & Abdominal infection $(n=15)$ & Pulmonary infection $(n=13)$ & $\mathrm{F}$ & P-value \\
\hline IL-4 (pg/l) & $327.84 \pm 24.51$ & $344.52 \pm 21.46$ & $355.75 \pm 28.54^{\mathrm{a}}$ & 7.122 & 0.002 \\
\hline IL-10 (ng/1) & $197.55 \pm 48.72$ & $209.24 \pm 52.63$ & $225.61 \pm 49.25$ & 1.605 & 0.209 \\
\hline $\mathrm{APN}(\mathrm{pg} / \mathrm{l})$ & $135.61 \pm 27.64$ & $126.91 \pm 23.97$ & $108.24 \pm 24.39^{\mathrm{a}}$ & 5.315 & 0.007 \\
\hline
\end{tabular}

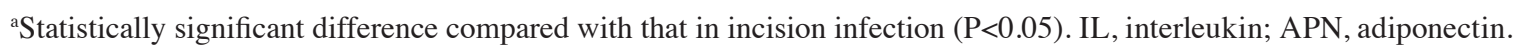

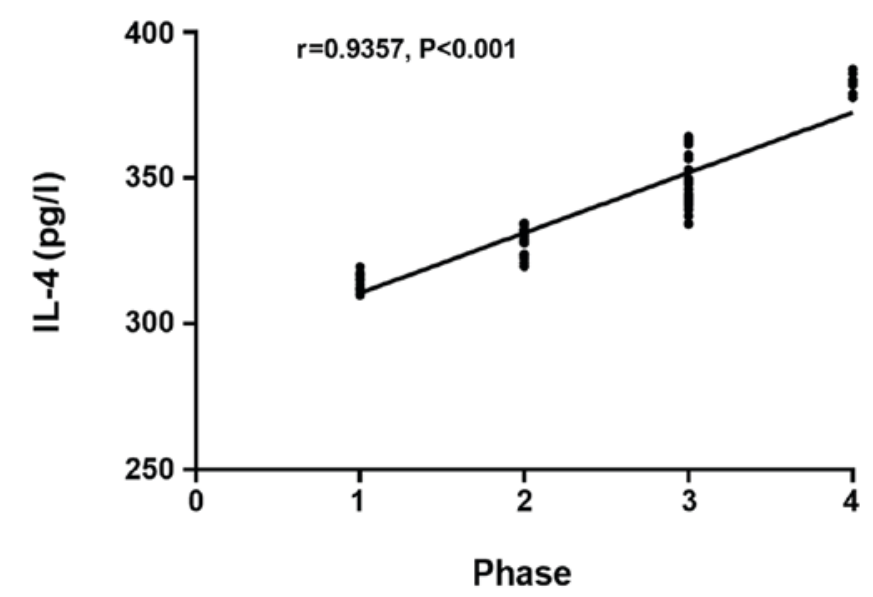

Figure 1. Correlation between IL-4 level at day 3 after surgery and stage of colorectal cancer in the infection group. Spearman's correlation analysis shows that the IL-4 level in patients with colorectal cancer at day 3 after surgery in the infection group has a significant positive correlation with the stage of colorectal cancer $(r=0.9357, \mathrm{P}<0.001)$. IL-4, interleukin-4.

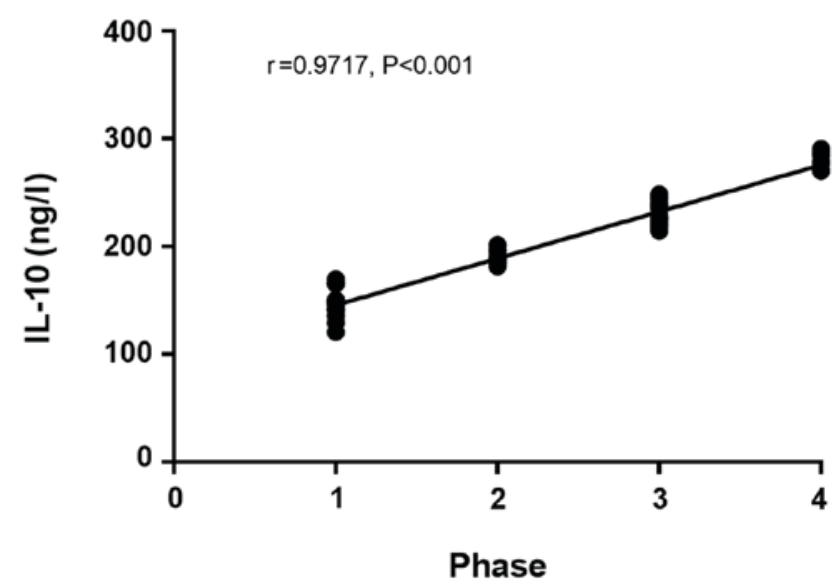

Figure 2. Correlation between IL-10 level at day 3 after surgery and stage of colorectal cancer in the infection group. Spearman's correlation analysis shows that the IL-10 level in patients with colorectal cancer at day 3 after surgery in the infection group has a significant positive correlation with the stage of colorectal cancer $(\mathrm{r}=0.9717, \mathrm{P}<0.001)$. IL-10, interleukin-10.

significant differences $(\mathrm{P}<0.05)$, and it was also lower in abdominal infection than that in incision infection without a statistically significant difference ( $\mathrm{P}>0.05$; Table III).

Correlation of serum $I L-4, I L-10$ and APN with stage of colorectal cancer in patients in infection group. The IL-4 and IL-10 levels in patients with colorectal cancer in the

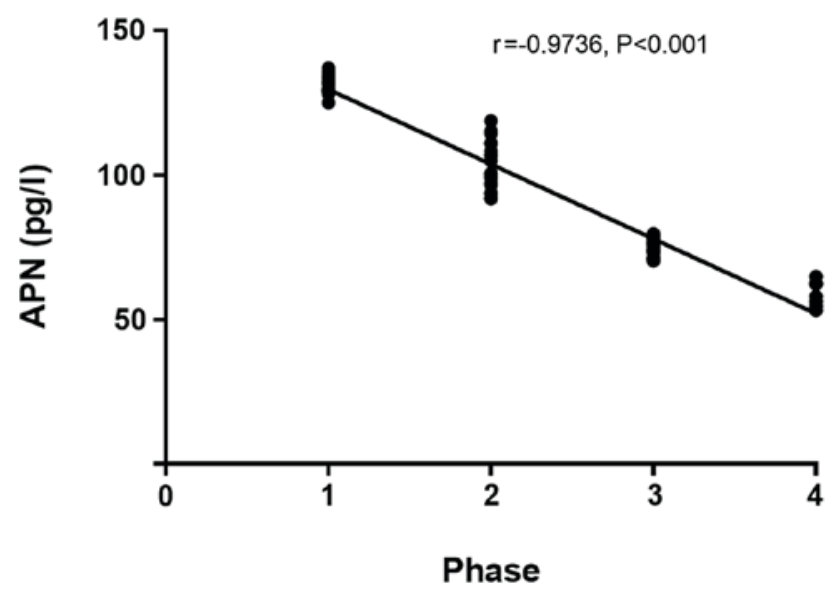

Figure 3. Correlation between APN level at day 3 after surgery and stage of colorectal cancer in the infection group. Spearman's correlation analysis reveals that the APN level in patients with colorectal cancer at day 3 after surgery in the infection group has a significant negative correlation with the stage of colorectal cancer $(r=-0.9736, \mathrm{P}<0.001)$. APN, adiponectin.

infection group at day 3 after surgery had a significant positive correlation with the stage of colorectal cancer $(r=0.9357$, $\mathrm{P}<0.001)(\mathrm{r}=0.9717, \mathrm{P}<0.001$; Figs. 1 and 2$)$, and the APN level in patients with colorectal cancer in the infection group at day 3 after surgery had a significant negative correlation with the stage of colorectal cancer ( $r=-0.9736, \mathrm{P}<0.001$; Fig. 3).

\section{Discussion}

The large intestine functions to absorb the liquid in the food debris and to make the food debris feces, which is an important component of the digestive system and the lower part of the digestive tract (10). At the same time, the large intestine also secretes mucin, so that the feces can be excreted easily and the intestinal wall can be protected from the mechanical damage (11). Colorectal cancer is divided into colon cancer and rectal cancer. There are no obvious symptoms in the early stage of colorectal cancer, and it has developed into advanced colorectal cancer if the lesion metastasizes to the stomach, liver and extra-territorial lymph nodes $(12,13)$. The position of colorectal cancer is low, so it can be diagnosed via digital rectal examination and colonoscopy. However, the large intestine is located deep in the pelvic cavity, so surgery is difficult and incomplete, and the postoperative recurrence rate and infection rate are extremely high (14). After radical surgery for colorectal cancer, pulmonary infection can occur in patients due to malnutrition, hypoproteinemia and anemia, abdominal 
infection can also be caused by increased abdominal pressure formed due to special functions of the large intestine, and there are various abdominal fats and the incision is prone to effusion, thus leading to incision infection (15-17).

After infection, the tissues in the patient's body will be stimulated by pain, trauma and stress, thereby releasing a large number of inflammatory factors. Both IL-4 and IL-10 are anti-inflammatory factors, which can exert an immunomodulatory effect in the large intestine through stimulating B cells, mastocytes, macrophages and T cells (18). IL-4 and IL-10 can inhibit the secretion of TNF, IL-1 and IL-6 and promote the body's immune response through downregulating inflammatory mediators (19). In the postoperative infection of patients with colorectal cancer, the activation process of Th1 and Th2 cells can be delayed under the combined action of IL-4 and IL-10, thereby enhancing the Th-type response and improving the body repair (20). APN, anti-inflammatory factor, is consumed due to the aggravation of inflammation in patients with postoperative infection of colorectal cancer, leading to significant decline in APN in the body. At the same time, the low-level APN can accelerate the occurrence of inflammatory response (21).

This study revealed that there were no differences in the expression levels of IL-4, IL-10 and APN between the two groups before surgery $(\mathrm{P}>0.05)$. The expression levels of IL-4 and IL-10 in the infection group were significantly higher than those in the non-infection group at day 3 after surgery $(\mathrm{P}<0.05)$, and they were higher in both groups after surgery than those before surgery $(\mathrm{P}<0.05)$, but the changes in expression levels in the infection group were more significant than those in the non-infection group. The expression level of APN in the infection group was lower than that in the non-infection group at day 3 after surgery $(\mathrm{P}<0.05)$, and it was lower in both groups after surgery than that before surgery $(\mathrm{P}<0.05)$, but the change in the expression level was more significant in the infection group than that in the non-infection group. The levels of anti-inflammatory factors IL-4 and IL-10 in the infection group were higher than those in the non-infection group, indicating that in the progression of postoperative infection of colorectal cancer, the anti-inflammatory effect of the patient's body is enhanced, and the decline in APN can strengthen the anti-inflammatory effect. The serum IL-4 level in pulmonary infection was higher than that in incision infection and abdominal infection $(\mathrm{P}<0.05)$, and it was also higher in abdominal infection than that in incision infection ( $P>0.05)$. The serum IL-10 level in pulmonary infection was higher than that in incision infection and abdominal infection $(\mathrm{P}<0.05)$, and it was also higher in abdominal infection than that in incision infection $(\mathrm{P}>0.05)$. The serum APN level in pulmonary infection was lower than that in incision infection and abdominal infection $(\mathrm{P}<0.05)$, and it was also lower in abdominal infection than that in incision infection $(\mathrm{P}>0.05)$. The expression levels of anti-inflammatory factors were different among different types of infections. Moreover, the IL-4 and IL-10 levels in patients with colorectal cancer in the infection group at day 3 after surgery had a significant positive correlation with the stage of colorectal cancer, and the APN level in patients with colorectal cancer in the infection group at day 3 after surgery had a significant negative correlation with the stage of colorectal cancer. It is reported (22) that the body immunity of patients with advanced colorectal cancer is poorer than that of patients with early colorectal cancer, which are consistent with the results in this study, and the expression levels of IL-4 and IL-10 in patients with advanced colorectal cancer were higher than those in patients with early colorectal cancer, while the expression level of APN was lower than that in patients with early colorectal cancer.

In this study, the sample size was small due to the limited medical resources in Xiangyang No. 1 People's Hospital Affiliated to Hubei University of Medicine, so there might be a certain contingency in the results. In the future, subjects in this study will be followed up for survey for a longer time, to obtain optimal results.

In conclusion, the serum IL-4, IL-10 and APN levels have a certain correlation with the presence or absence of postoperative infection of colorectal cancer, the type of infection and the stage of colorectal cancer, which are worthy of clinical promotion.

\section{Acknowledgements}

Not applicable.

\section{Funding}

No funding was received.

\section{Availability of data and materials}

The datasets used and/or analyzed during the current study are available from the corresponding author on reasonable request.

\section{Authors' contributions}

JH and ZW performed ELISA. SZ analyzed the general data of patients and revised the manuscript. All authors read and approved the final manuscript.

\section{Ethics approval and consent to participate}

This study was approved by the Ethics Committee of Xiangyang No. 1 People's Hospital, Hubei University of Medicine (Xiangyang, China). Patients who participated in this study, signed the informed consent and had complete clinical data.

\section{Patient consent for publication}

Not applicable.

\section{Competing interests}

The authors declare that they have no competing interests.

\section{References}

1. Siegel RL, Miller KD, Fedewa SA, Ahnen DJ, Meester RGS, Barzi A and Jemal A: Colorectal cancer statistics, 2017. CA Cancer J Clin 67: 177-193, 2017. 
2. Arnold M, Sierra MS, Laversanne M, Soerjomataram I, Jemal A and Bray F: Global patterns and trends in colorectal cancer incidence and mortality. Gut 66: 683-691, 2017.

3. Bibbins-Domingo K, Grossman DC, Curry SJ, Davidson KW, Epling JW Jr, García FAR, Gillman MW, Harper DM, Kemper AR, Krist AH, et al; US Preventive Services Task Force: Screening for colorectal cancer: US preventive services task force recommendation statement. JAMA 315: 2564-2575, 2016.

4. Lin JS, Piper MA, Perdue LA, Rutter CM, Webber EM O'Connor E, Smith N and Whitlock EP: Screening for colorectal cancer: updated evidence report and systematic review for the US preventive services task force. JAMA 315: 2576-2594, 2016.

5. Favoriti P, Carbone G, Greco M, Pirozzi F, Pirozzi RE and Corcione F: Worldwide burden of colorectal cancer: a review. Updates Surg 68: 7-11, 2016.

6. McSorley ST, Watt DG, Horgan PG and McMillan DC: Postoperative systemic inflammatory response, complication severity, and survival following surgery for colorectal cancer. Ann Surg Oncol 23: 2832-2840, 2016.

7. Tei M, Wakasugi M, Kishi K, Tanemura M and Akamatsu H: Incidence and risk factors of postoperative delirium in elderly patients who underwent laparoscopic surgery for colorectal cancer. Int J Colorectal Dis 31: 67-73, 2016.

8. Sakellariou S, Fragkou P, Levidou G, Gargalionis AN, Piperi C, Dalagiorgou G, Adamopoulos C, Saetta A, Agrogiannis G, Theohari I, et al: Clinical significance of AGE-RAGE axis in colorectal cancer: associations with glyoxalase-I, adiponectin receptor expression and prognosis. BMC Cancer 16: 174, 2016.

9. Lasry A, Zinger A and Ben-Neriah Y: Inflammatory networks underlying colorectal cancer. Nat Immunol 17: 230-240, 2016.

10. Kojima M, Ikeda K, Saito N, Sakuyama N, Koushi K, Kawano S, Watanabe T, Sugihara K, Ito M and Ochiai A: Neuroendocrine tumors of the large intestine: clinicopathological features and predictive factors of lymph node metastasis. Front Oncol 6: 173, 2016.

11. Bettington M, Walker N, Rosty C, Brown I, Clouston A, McKeone D, Pearson SA, Klein K, Leggett B and Whitehall V: Serrated tubulovillous adenoma of the large intestine. Histopathology 68: 578-587, 2016.

12. Sinicrope FA, Okamoto K, Kasi PM and Kawakami H: Molecular biomarkers in the personalized treatment of colorectal cancer. Clin Gastroenterol Hepatol 14: 651-658, 2016.
13. Mármol I, Sánchez-de-Diego C, Pradilla Dieste A, Cerrada E and Rodriguez Yoldi MJ: Colorectal carcinoma: a general overview and future perspectives in colorectal cancer. Int J Mol Sci 18: 197, 2017.

14. Tariq K and Ghias K: Colorectal cancer carcinogenesis: a review of mechanisms. Cancer Biol Med 13: 120-135, 2016.

15. Koelzer VH, Zlobec I and Lugli A: Tumor budding in colorectal cancer-ready for diagnostic practice? Hum Pathol 47: 4-19, 2016.

16. Tanaka K, Kumamoto T, Nojiri K, Matsuyama R, Takeda K and Endo I: Impact of postoperative morbidity on long-term survival after resection for colorectal liver metastases. Ann Surg Oncol 23 (Suppl 5): 929-937, 2016.

17. Mokutani Y, Mizushima T, Yamasaki M, Rakugi H, Doki Y and Mori M: Prediction of postoperative complications following elective surgery in elderly patients with colorectal cancer using the comprehensive geriatric assessment. Dig Surg 33: 470-477, 2016.

18. Park JH, Watt DG, Roxburgh CS, Horgan PG and McMillan DC: Colorectal cancer, systemic inflammation, and outcome: staging the tumor and staging the host. Ann Surg 263: 326-336, 2016.

19. Landy J, Ronde E, English N, Clark SK, Hart AL, Knight SC, Ciclitira PJ and Al-Hassi HO: Tight junctions in inflammatory bowel diseases and inflammatory bowel disease associated colorectal cancer. World J Gastroenterol 22: 3117-3126, 2016.

20. Brennan CA and Garrett WS: Gut microbiota, inflammation, and colorectal cancer. Annu Rev Microbiol 70: 395-411, 2016.

21. Passardi A, Scarpi E, Cavanna L, Dall'Agata M, Tassinari D, Leo S, Bernardini I, Gelsomino F, Tamberi S, Brandes AA, et al: Inflammatory indexes as predictors of prognosis and bevacizumab efficacy in patients with metastatic colorectal cancer. Oncotarget 7: 33210-33219, 2016.

22. Moriarity A, O'Sullivan J, Kennedy J, Mehigan B and McCormick P: Current targeted therapies in the treatment of advanced colorectal cancer: A review. Ther Adv Med Oncol 8: 276-293, 2016

This work is licensed under a Creative Commons Attribution-NonCommercial-NoDerivatives 4.0 International (CC BY-NC-ND 4.0) License. 